\title{
Sadharanikaran Model vs Mathematical Model: Analyzing their Relevance in Context of Social Media
}

Priya Shaw

\begin{abstract}
The communication process varies according to cultural, political, economic, social, psychological, socio-economic factors from one country to another. The term 'communication', however, has been accepted globally though the pattern of communication differs from one society to another. Communication scientists have tried to define communication in a particular context of a particular society. In this study, therefore, Sadharanikaran model from the Eastern perspective and Shannon and Weaver model from the Western perspective have been chosen to study the difference in the pattern of communication. The elements of the models have been studied along with the context of technological communication with particular reference to social media such as Facebook, WhatsApp and Skype.
\end{abstract}

Keywords: Sadharanikaran Model of Communication, Mathematical Model of Communication, Social Media.

\section{Introduction}

The term Sadharanikaran has been rooted in Natyasastra of Bharata. Rasa Sutra in Natyasastra is the basic concept on which the theory of Sadharanikaran has been developed. There have been attempt to extend its history up to the Vedic 
period (Adhikary, 2007a, p.108) but scholars widely believe that Bhattayanka introduced the concept of Sadharanikaran (Vatsyanan, 1996, p.146). He did so while explaining the rasa sutra of Bharata's Natyasastra. The term Sadharanikaran has been derived from the Sanskrit word Sadharan, and has been translated into English as "generalized presentation" (Vedantatirtha, 1936, p.35)" simplification" (Yadava,1998, p.187) and "universalization" (Dissanayake, 2006, p.4).

Dr. Nirmal Mani Adhikary gave the Sadharanikaran Model of Communication (SMC) in 2003 and it was revised in 2010. According to the model the elements of communication are:

1. Sahridaya (Preshaka- Sender and Prapaka- Receiver)

2. Bhava (Moods and Emotions)

3. Abhivyanjana (Expression and encoding)

4. Sandesha (Message or Information)

5. Sarani (Channel)

6. Rasaswadana (Firstly receiving, decoding and interpreting the message and finally achieving the rasa)

7. Doshas (Noise)

8. Sandharbha (Context)

9. Pratikriya (Feedback)

Sahridayas are those who are having "common Sympathetic heart" (Yadava, 1998, p.188). Preshaka (Sender) and Prapaka (Receiver) must attain sahridaya in order to have a coequal emotion towards the message. In a society where asymmetrical relations exist, there sahridayata is the only way of two-way communication and mutual understanding. Thus, sahridayata can be achieved irrespective of castes, languages, cultures and religious practices and this type of 
communication process qualifies to be considered as Sadharanikaran.

Sahridaya preshaka (sender) who has bhava (Moods and Emotions or Thought) in mind is the initiator of the process. The Sender has to pass the process of abhivyanjana for encoding the bhava in a perceivable form. It is the Sahridaya prapaka with whom the bhavas are to be shared. He must go through the process of rasaswadana. The prapaka is engaged in receiving, decoding and interpreting the message to achieve the rasa i.e. the essence of aesthetic enjoyment.

The position of Sahridaya preshaka and prapaka is not static. Both parties are engaged in the process of abhivyanjana and rasaswadana. A successful sadharanikaran leads toward the universalization. If there is no bhava there is no need of communication. The sandesha which the preshaka wants to convey to the prapaka must arouses bhavas and go through the process of abhivyanjana and the sandesa will be received by the prapaka after going through the process of rasaswadana and at the conclusion sahridayata will be attained.

For transmission of sandesha, sarani (channel or medium) is required through which sandesha travels across space. The message sent by the sender cannot reach the receiver without the channel or medium. The channels may be natural such as hearing, touching, visual as well as mechanical such as telephone, radio, computer etc. perfect communication is impossible to achieve. There are continuous forces at work, doshas or noises which tend to distort the message and leads to miscommunication. If we look at Hindu poetics the concept of rasa-bhanga (disruption in rasaswadana) is there. There may be many causes of this. For instance, a mismatch between 
sender and receiver. The noises are like semantic, mechanical and environmental.

For effective communication sandarbha (context) is needed. It helps the prapaka to decode the sandesha which has been sent by the preshaka. The effectiveness of any message depends on communication environment. Same message may have different meaning in different context. The importance of context is such that the meaning could be provided to the receiver even if the sender is not identified to the receiver. Thus, due to context a text can retain to its objective meaning".

Pratikriya refers to the responses of the receiver after receiving the message. It is the process of feedback which allows the receiver to have an active role in communication process. Sadharanikaran demands sahridayata in which both sender and receiver are taken into consideration. It is not that feedback is always affirming. However, it makes the communication process ongoing. In every sphere, feedback is not necessary. If the sender and receiver are able to understand and experience the same equally, there no feedback is required. ${ }^{1}$

In 1949 an engineer and researcher at Bell Laboratories, named Claude E. Shannon, founded an information theory based on mathematical theories which was about signal transmission with maximum telephone line capacity and minimum distortion. For the first time, he introduced the qualitative and quantitative model of communication as a

\footnotetext{
${ }^{1}$ Dr. Nirmal Mani Adhikary 'Theory and Practice of Communication- Bharata Muni' 2014
} 
statistical process underlying information theory. The aim of Shannon was signal transmission from source to destination through transmitter and receiver across the channel with minimal interference or error. The information theory initially developed to separate noise from the signals carrying the information. Later on, Weaver extended and applied Shannon's information theory for different kinds of communication. While Shannon was focused on engineering aspect of his theory, Weaver developed the philosophical aspects of this theory related to human communication. Shannon and Weaver model of communication has 7 main elements which are:

1. Information source

2. Transmitter (encoder)

3. Channel

4. Noise

5. Signal

6. Receiver (decoder)

7. Message

8. Destination

The information source, selects a desired message out of a set of possible messages. The selected message may consist of written or spoken words, or of pictures, music, etc. The transmitter changes this message into the signal which is actually sent over the communication channel from the transmitter to the receiver. The receiver is a sort of inverse transmitter, changing the transmitted signal back into a message, and handing this message on to the destination. In the process of being transmitted, it is unfortunately characteristic that certain things are added to the signal which 
was not intended by the information source. These unwanted additions may be distortions of sound. All of these changes in the transmitted signal are called noise. The researchers identify three levels of problem in communication those are technical problem, semantic problem, effectiveness problems. $^{2}$

Social media refers to the use of web-based and mobile technologies to turn communication into an interactive dialogue. In the words of Andreas Kaplan and Michael Haenlein, social media is "a group of Internet-based applications that build on the ideological and technological foundations of Web 2.0, and that allow the creation and exchange of user-generated content." Kaplan and Haenlein created a classification scheme for different social media types in their Business Horizons article published in 2010. According to Kaplan and Haenlein there are six different types of social media: collaborative projects (e.g. Wikipedia), blogs and microblogs (e.g. Twitter), content communities (e.g. YouTube), social networking sites (e.g. Facebook), virtual game worlds (e.g. World of Warcraft) and virtual social worlds (e.g. Second Life). Technologies include: blogs, picture-sharing, vlogs, wall-postings, email, instant messaging, music-sharing, crowdsourcing and voice over IP, to name a few. Many of these social media services can be integrated via social network aggregation platforms. Currie (as cited by Hysenlika, 2012) defined social media as "the various electronic tools, technologies, and

\footnotetext{
${ }^{2}$ http://waste.informatik.huberlin.de/Lehre/ss11/SE Kybernetik/reader/weaver.pdf
} 
applications that facilitate interactive communication and content exchange, allowing the user to move back and forth easily between the roles of the audience and content producers". Social media is becoming such an integral part of business operations that its use is now commonplace for product launches, developing consumer loyalty and sharing. Currie (as cited by Hysenlika, 2012) defined social media as "the various electronic tools, technologies, and applications that facilitate interactive communication and content exchange, allowing the user to move back and forth easily between the roles of the audience and content producers". Social media is becoming such an integral part of business operations that its use is now commonplace for product launches, developing consumer loyalty and sharing news (Walaski, 2013).

\section{Review of Literature}

Adhikary (2003) has introduced an article on Sadharanikaran Model of Communication. The Sadharanikaran model illustrates how the communicating parties interact through the process of sadharanikaran to attain saharidayata (commonness or oneness). The model "offers an explanation of how successful communication is possible in Hindu society where complex hierarchies of castes, languages, cultures and religious practices are prevalent" (Adhikary, 2008a, p. 67). The model has been observed as a representation of communication process from Hindu perspective. The model is a non-linear one. Importance has been given on the interrelationship between communicating parties and encoding and decoding are fundamental activities of this 
model. The model also deals with physical, mental and spiritual level and the main aim remains in achieving commonness.

Adhikary (2008) wrote an article to study the fundamental differences between the Sadharanikaran model and Aristotle's model of communication. The main aim is to bring out the concepts of communication process which have envisioned in two different models from the East and the West. The general objective here is to comprehend the fundamental differences between the concepts of communication process in Aristotelian and Sadharanikaran view. The specific objectives include having comparative understanding of the concept of communication process from Aristotelian and Sadharanikaran perspectives in terms of structure and scope of two models, the human relationships in the process and the goal of communication. To conduct the study, purposively the models have been chosen.

Barua (2012) has researched on the effectiveness on social media as a tool of communication. The aim was to study the impact, usefulness and potential of social media. The study has based on qualitative and quantitative analysis of the role and importance of social media as a tool of effective communication. Survey method and observation method were used to gather data from 200 respondents from Guwahati. Respondents agreed that social media is a good platform to communicate with large number of people in limited time.

Choudhury and Bhattyacharya (2014) have conducted their work on communication from Indian perspective in which special reference was given to Natyasastra. The main importance has given to sadharanikaran which is an Indian 
perspective of communication. Dramatic representation of Sadharanikaran process enhances the relation between communicator and communicates. Sadharanikaran enhances intra personal communication too which has been described here.

\section{Reason to Conduct the Study}

Sadharanikaran model is important to understand the eastern perspective of communication whereas Shannon and Weaver model is to understand western perspective. Both models are practical enough to be fitted in present scenario. Human communication is the base of SMC and human communication with the help of technology is the base for Shannon and Weaver model. So, it is a sort of curiosity from my side to know what possible conclusion can be drawn by putting these two opposite models together in present situation and how these two models differ in their elements. The advancement of communication is blessed with advanced technology. Social media is a platform to communicate with many people at a single time. Relevance of these models in social media platform is also a matter of concern. Whether or not the elements of human communication models are equally applied on technology based media is an important area to study. 


\section{Objectives:}

- To distinguish the elements of Sadharanikaran Model of Communication and Shannon and Weaver Mathematical Model of Communication

- To analyze the relevance of these two models in the context of social media.

\section{Research Methodology}

- Purposively Sadharanikaran model from eastern perspective and Shannon and Weaver Mathematical Model of Communication from western perspective have been selected

- Basically, theoretical approach has been adopted and the study is based on secondary data though the conclusion which has been drawn is an exploratory one.

\section{A Comparative Analysis between the Two Models:}

Let us now make a comparative analysis between the two models.

- The Sadharanikaran model is a non-linear one. It is resulting in two-way communication. In this case, both inter and intra personal communication is possible whereas Mathematical Model of Communication is a 
linear one. Here, only one aspect of communication has been notified. The whole communication process is controlled by the sender only.

- Sadharanikaran model is stressed mostly face to face communication whereas Mathematical Model of Communication focused on mechanical aspect of communication.

- In Sadharanikaran model the sandesha of the preshaka is filled with bhavas and it helps the sender to pass through the process of abhivyajana whereas in Mathematical Model of Communication there is no discussion on bhavas. The model is a technical one. No emotion is attached in this communication.

- Sadharanikaran model illustrates the Hindu perspective of communication..A dissertation (Adhikary, 2003), taking insights from both Natyashastra and Vakyapadiya and integrating them, presents a unique communication model - the Sadharanikaran model of communication, which was the first ever model of communication in diagrammatic form proposed from Hindu perspective. On the other hand, Mathematical Model of Communication depicted the Western perspective of communication as the model does not have reflection of classical Sanskrit text.

- In Sadharanikaran, the receiver must go through the process of rasaswadana in order to achieve the true essence of the sandesha and to understand the rasa behind sending the message. But in Mathematical Model of Communication a transmitter is decoding the message and reaching to the destination; no rasa is associated with it.

- In Sadharanikaran model, the emphasis has also been given on sandharbha i.e. the base on which communication will be started. The source of 
communication is present there. In Mathematical Model of Communication, the concept of context is missing. The model has not mentioned what will be the source of communication.

- In Sadharaikaran the main motto is to achieve the oneness or commonness and after achieving sahridayata the communication will be proven successful. Again, in case of Mathematical Model of Communication there is no emotional connection between the sender and the receiver so there is no question of oneness is coming into account. The inter-relationship between two parties are top most important. And in Sadharanikaran oneness can be achieved irrespective of asymmetrical hierarchies. In Mathematical Model of Communication, it is not clear what will be the hierarchy between the sender and the receiver whether it will be equal or asymmetrical.

- Sadharanikaran model is also talked about pratikriya means what will be the feedback of the receiver after receiving the message which may lead to further communication. To have a successful communication feedback is needed. But in Mathematical Model of Communication no possibility of feedback is available. It is impossible to measure the reaction of the receiver after receiving the message. Somehow, the road of further communication has been blocked. Through the process of pratikriya the effectiveness of the communication can also be measured. 


\begin{tabular}{|l|l|}
\hline Sadharanikaran Model & Mathematical Model \\
\hline $\begin{array}{l}\text { Sender (Preshaka) } \\
\text { (Sahridaya) }\end{array}$ & Source \\
\hline $\begin{array}{l}\text { Moods or emotions } \\
\text { (Bhava) }\end{array}$ & -------------------- \\
\hline $\begin{array}{l}\text { Expression or encoding } \\
\text { (Abhivyanjana) }\end{array}$ & Transmitter (encoder) \\
\hline $\begin{array}{l}\text { Message or information } \\
\text { (Sandesha) }\end{array}$ & Message \\
\hline $\begin{array}{l}\text { Communication code } \\
\text { (Sanketa) }\end{array}$ & ------------------- \\
\hline------------------ \\
\hline Channel (Sarani) & Signal \\
\hline $\begin{array}{l}\text { Receiving, decoding and } \\
\text { interpreting the message } \\
\text { and finally achieving the } \\
\text { rasa (Rasaswadana) }\end{array}$ & Receiver (decoder) \\
\hline $\begin{array}{l}\text { Aesthetic enjoyment } \\
\text { (Rasa) }\end{array}$ & -------------------- \\
\hline Noise (Doshas) & Noise \\
\hline Context (Sandharbha) & ------------------- \\
\hline $\begin{array}{l}\text { Receiver (Prapaka) } \\
\text { (Sahridaya) }\end{array}$ & Destination \\
\hline Feedback (Pratikriya) & Feedback ${ }^{3}$ \\
\hline
\end{tabular}




\section{Observation}

On the basis of the above discussion, linear, one-way communication processes have been described in the Shannon and Weaver's mathematical Model of Communication. Mainly, Western aspect of communication has taken into consideration. No possibility of feedback was available before 1970. The model was expanded with the feedback by the theoretician Melvin DeFleur, adding further parts and introducing the term "meaning". This is transformed by the source into "information", sent by the transmitter into the channel and again changed by the receiver to the destination. It is the feedback that plays a significant role for checking the quality of information service. Sadharanikaran model of communication has described all possibilities which occur at the beginning and stretch up to the end of a successful communication process. The actual essence of communication is visible in Sadharanikaran model of communication which leads to the inner satisfaction which one can hold after having a proper and complete communication.

\footnotetext{
${ }^{3}$ There was no provision in the original model for feedback (reaction from the receiver). Feedback enables speakers to adjust their performance to the needs and responses of their audience. A 'feedback loop' was added by later theorists, but the model remains linear. (http://visualmemory.co.uk/daniel/Documents/short/trans.html)
} 


\section{Critical Analysis of Models}

Feedback is essential if one wants to keep the communication on and it is associated with the receiver's part. In these models, it is not mention that feedback may also associate with the receiver's mood, interest and attitude towards the topic of communication. If a receiver is unable to identify himself with the sender's idea at that time, no "pratikriya" will be available from the receiver's side and there is a possible chance of having fool stop over the communication. Interest is also an integral part for molding feedback such as opinion, suggestion and so on. May be the source is delivering an important message but the receiver does not have any interest to listen that; the process of feedback will be hampered.

The concept of mood must also be taken into consideration while discussing about feedback. If throw of a message is in temperament or in anger the receiver may refuse to listen the message or may not pay heed towards the message or may disconnect the telephone line again feedback will be in risk. In this case, there will be a serious jerk in between sender and receiver as well as in the whole communication process. Here, the hierarchy between the two must take into account. If the flow of the message is from top to down; the receiver is forced to listen that whether he is interested or not. Here the chance of feedback is also not available.

Again, in case of interpersonal or group communication the possibility of feedback is available but in case of mass communication feedback is not possible. According to Shannon and Weaver Model, one information source and one destination is necessary for completion of the communication 
process. In case of telephonic communication, there may remain more than single sender and receiver.

In case of Sadharanikaran model of communication, the emphasis has also given on 'sandarbha' which depicts that both the parties must be aware of the context on which the communication will be proceed further and the receiver will pass through the process of 'rasaswadana' in order to achieve the rasa which has been included at the time of constructing the message. It is clear that both are aware of the context of the communication but the receiver may have no opinion on that context, in that case rasaswadana is not possible for the receiver and no feedback will be available which results an abrupt discontinuation during the communication.

\section{Relevance of the Models in Technological Context}

From the ancient time, there is a need of communication whether in oral or written format. Interpersonal communication plays a major role in order to have a direct and instant response on certain context. If we judge in today's context, usually two types of interpersonal communication are there:

1. Face to face communication

2. One-to-one communication with the help of technology

Society is fast changing and in every moment an old technology is being taken up by new and advanced one. Everyday new applications are launched for improving 
communication. I would like to judge the relevance of Sadharanikaran model of communication and Telephonic model in the context of social media communication taking Facebook, WhatsApp, Skype in this account.

Social media is flexible enough to communicate with a large number of people at once in a limited time. Both interpersonal and group communication can be done through it. Sadharanikaran model of communication talks about only face to face communication which can be attained only between sahridayas. We use social networking sites for interpersonal communication but in a written format. Here, sender is having time flexibility to response. The possibility of feedback is entirely depending on the receiver's mood while there is a time flexibility the receiver may response after a long time or may be in between he leave the conversation. The process of abhivyanjana and rasaswadana may not be fully achieved here.

The bhava which the sender has included while framing the message may not be decoded by the receiver properly as they are not present there physically. Interpersonal communication at a distance leads to less chance of arousing the same feeling between the sender and receiver. It is very clear though social media is providing opportunity of interpersonal communication but the actual motto of sadharanikaran model of communication is not followed here fully. Again, the communication with video calling will provide the highest possibility to provoke the same mood and achieving the rasa and result of a proper communication.

Shannon and Weaver had brought technology for interpersonal communication in its account. Today, simple 
telephonic communication has been replaced with iPhone, tablets, and laptop which have given new shape of interpersonal communication. All these gadgets give us the equal chance for having interpersonal communication with the help of social networking sites. The gadgets are improving the possibility of communication but again the feedback depends on the receiver's mood entirely. All the elements of Shannon and Weaver model are present here. Semantic and technological error are also present in communication while is being done through social media.

\section{Conclusion}

Sadharanikaran model of communication talks about moods, abhivyanjana and rasaswadana which clearly depicts that emotional attachments are necessary whereas in Shannon and Weaver model the focus has only been given on communication part no emotional attachments are there between sender and receiver. Both models are having different perspective of depicting communication.

Sadharanikaran model of communication entirely speaks about intra and face to face interpersonal communication. To achieve rasa it is necessary to have a continuation of conversation. In case of theatre performance individual artist try to connect him/herself with individual audience in order to have a complete flow of emotions and feeling which results achieving the same rasa. Sometimes, the individual goes into the process of interpersonal communication where he/she relates his situation with the character. 
Shannon and Weaver model of communication is technology oriented. As in, sadharanikaran model focus is on face to face communication; here encoder and decoder are human beings. In case of technology, encoder and decoder is transmitter. The picture is clearly understood whether we are in a telephonic communication or web communication. As a conclusion both models are relevant according to their situation to achieve the best possible communication.

\section{References}

Adhikary, N.M. (2014). Indian Tradition of Communication-1 Theory and Practice of Communication -Bharata Muni, MakhanlalChaturbedi National University of Journalism and Communication, Bhopal.

Adhikary, N.M. (2013). Communication theory and classical Sanskrit texts. Rural Aurora, 2, 112-125.

Adhikary, N. (2009). An Introduction of Sadharanikaran Model of Communication, Bodhi: An Interdisciplinary Journal, 3(1) ISSN 2091-0479.pp 69-91.

Adhikary, N. (2008). The SadharanikaranModel and Aristotle's Model of Communication: A Comparative Study, Bodhi: An Interdisciplinary Journal, 2(1)ISSN 2091-0479.

Adhikary, N. M. (2008a). Communication, media and journalism: An integrated study. Kathmandu:

PrashantiPrakashan. 
Adhikary, N. M. (2007a). Sancharko Hindu awadharanatmakadhyayan. In N. M. Adhikary, Sanchar shodhara media paryavekshan (pp. 93-138). Kathmandu: PrashantiPustakBhand.

Adhikary, N. M. (2003). Hindu awadharanamasancharprakriya [Communication in Hindu concept]. A dissertation presented to Purvanchal University, Nepal in the partial fulfillment of the requirements for the Degree of Master of Arts in Mass Communication and Journalism.

Baruah, D , T. (2012). Effectiveness of Social Media as a Tool of Communication and Its Potential for Technology Enabled Connections: A Micro Level Study, International Journal of Scientific and Research Publications, 2(5) ISSN 2250-3153.

Choudhury, L, B, and Bhattacharya, K, K. (2014).

Communication from Indian Perspective with Special Reference to Natyasastra. DEV SANSKRITI:

Interdisciplinary International Journal, vol.04(ISSN:22790578). pp 62-72

Datta, R. (2013). Philosophizing Communication: A Reading of Natyasastra, Global Media Journal- Indian Edition, 4(1) ISSN 2249-5835.

Derani,N, E, S, and Naidu, P. (2016). The Impact of Utilizing Social Media as a Communication Platform During a Crisis Within the Oil Industry, Procedia Economics and Finance, 35 ISSN 2212-5671.pp 650 - 658. 
Hysenlika, V., 2012. Communicating During an Organizational Crisis: Using Facebook as a Relationship Management Tool (Doctoral Dissertation, University of South Florida).

Kaplan, M, A, and Haenlein, M. (2012). Social Media: back to the roots and back to the future, Journal of Systems and Information Technology Vol. 14 No. 2, 2012. pp. 101-104. Emerald Group Publishing Limited 1328-7265 DOI $10.1108 / 13287261211232126$.

Weaver, and Warren. (1949). 'Recent Contributions to the Mathematical Theory of Communication'. Retrieved from http://waste.informatik.huberlin.de/Lehre/ss11/SE Kybernetik/reader/weaver.pdf

Walaski, P., 2013. Social Media. Professional Safety, 58(4), 40-49.

http://search.proquest.com/docview/1331594111?accounti $\mathrm{d}=13380$.

Wimmer, R. D., and Dominick, J. R. (2003). Mass media research: An introduction. Belmont, CA: Wadsworth.

Yadava, J. S. (1998). Communication research in India: Some reflections. In J. S. Yadava and P. Mathur (Eds.), Issues in mass communication: The basic concepts (pp. 177-

195). New Delhi: IIMC.

Retrieved http://visualmemory.co.uk/daniel/Documents/short/trans.html 
Retrieved

https://islmblogblog.files.wordpress.com/2016/05/assignm ent3shannonandweavermodel.pdf

The author is an Assistant Professor at the Department of Media and Communication, Centurion University of Technology and Management.

Email: priyashaw05@gmail.com 